\title{
Multiple electrically tunable parametric resonances in a capacitively coupled electromechanical resonator for broadband energy harvesting
}

\author{
Sushruta Surappa ${ }^{1} \oplus$, Tuna Erdogan ${ }^{2}$ and $\mathrm{F}$ Levent Degertekin ${ }^{1, *}$ \\ ${ }^{1}$ G. W. Woodruff School of Mechanical Engineering, Georgia Institute of Technology, Atlanta, \\ GA 30332, United States of America \\ ${ }^{2}$ Department of Electrical and Electronics Engineering, Ihsan Dogramaci Bilkent University, Ankara, \\ Turkey \\ E-mail: levent.degertekin@me.gatech.edu
}

Received 4 January 2021, revised 5 February 2021

Accepted for publication 25 February 2021

Published 12 March 2021

\begin{abstract}
Parametric excitation (PE) has widely been employed as a method of mechanical pre-amplification in nonlinear vibration energy harvesting systems. However, despite their advantages, most current PE systems are limited to degenerate parametric operation within a narrow frequency band around the primary instability tongue. In this paper, we simulate and experimentally demonstrate a parametrically driven capacitive electromechanical resonator having multiple electrical degrees of freedom. Multiple modes allow for several frequency bands in which the electrical resonator is driven into nondegenerate (combination) parametric resonance (PR) in addition to degenerate resonance, thereby enabling operation over a broader range of frequencies while maintaining the same mechanical footprint. These frequency bands and PR thresholds are tunable by simply changing the electrical circuit parameters and PR can be achieved in the presence of high mechanical damping making the method more adaptable than purely mechanical approaches. Experimental results are extended by simulations indicating that proper selection of operating parameters can enable the merging of instability tongues to produce a broadband region of PR for elastic wave energy harvesting thereby obtaining superior performance when compared to an equivalent single degree of freedom PE energy harvester.
\end{abstract}

Supplementary material for this article is available online

Keywords: tunable resonators, MEMS, nonlinear systems, parametric excitation, energy harvester

(Some figures may appear in colour only in the online journal)

\section{Introduction}

Vibration energy harvesting by means of parametrically excited (PE) systems has gained increased attention in recent years [1-5]. Unlike linear resonant systems, the vibrational

* Author to whom any correspondence should be addressed. response of a PE system is independent of the linear damping and only limited by nonlinearities at large amplitudes [6]. This has enabled the use of PE as a means of mechanically amplifying the vibrational motion of the energy harvester. Primary parametric resonance (PR) is obtained by periodically modulating a system parameter such as the stiffness at approximately twice the natural frequency of the system. When the 
modulation exceeds a damping dependent threshold, a sharp increase in the system amplitude response is observed and the vibration is converted to electrical energy by means of piezoelectric [7-9], electromagnetic [6, 10, 11] and other [12] transduction methods.

PE energy harvesters have previously been shown to outperform their linear counterparts in terms of bandwidth and power density $[6,8]$, however, this class of energy harvesters is still limited by two major drawbacks - a high initiation threshold and narrow bandwidth. The damping dependent threshold for PR in a purely mechanical system can sometimes place an impractical restriction on the minimum excitation level required for effectively harvesting energy from ambient vibrations, as the system is essentialy rendered unusable for excitation levels below this threshold. Researchers have tried to overcome this limitation by combining direct and PEs in the harvesting system, as well as developing designs that reduce the initiation threshold of the PE energy harvester $[7,13,14]$.

The instability tongue in which a PE energy harvester is driven into PR is limited to a narrow band around two times the natural frequency of the system and this places a significant restriction on the operational bandwidth of the harvester. Different approaches have been proposed to overcome this limitation, including using systems with multiple mechanical degrees of freedom (DOF) [15-19]. However, such an approach leads to an increase in the size of the energy harvester without a significant increase in the energy density. Attempts have also been made to broaden the operation bandwidth by exploiting the higher order PRs of a single mechanical resonator $[20,21]$. While this can be achieved in a small footprint, it is well known that higher order PRs are difficult to excite and have narrower instability tongues [20] when compared to the first order PR, making them highly impractical for real world energy harvesting applications. More recent developments include the use of clever designs to modify the nonlinearity of the system and improve the bandwidth of the energy harvester [10, 22, 23]. However, such devices usually involve complex design and are not trivial to realize at the micro scale.

Recent advances in PE transducers for wireless power transfer [24], energy harvesting [25] and acoustic sensing [26] has led to the development of mechanically driven electrical parametric resonators. The capacitive parametric ultrasonic transducer (CPUT) is one such system comprised of a membrane-based time varying parallel plate capacitor that forms part of a resistor-inductor-capacitor (RLC) resonator [27]. Mechanically exciting the membrane around two times the natural frequency of the RLC circuit causes the electrical resonator to be driven into $\mathrm{PR}$, resulting in the conversion of the input mechanical energy into an electrical output without the need for a bias voltage or a secondary transducer. Additionally, the forcing threshold for PR in the CPUT is primarily dependent on the damping in the electrical circuit [26]. Therefore it can be operated in lossy environments with damped mechanical characteristics and with electrically controlled initiation, thereby lifting a significant limitation faced by purely mechanical PE systems.
In this paper, we propose the use of multiple electrical DOF $(\mathrm{MDOF})^{3}$ to both excite and accurately control the location of degenerate and nondegenerate (combination) PRs while using a single mechanical resonator. The operation of such a system is first simulated using a 1D lumped model in Simulink and experimentally verified by ultrasonically exciting a capacitive micro electro mechanical systems (MEMS) resonator with two electrical DOF into multiple regions of PR in a fluid medium. Tunability of the system just by changing electrical circuit elements is also shown experimentally. The utility and scalability of such an implementation to practical low-frequency elastic wave energy harvesting is then demonstrated by designing and simulating a 2-DOF PE capacitive energy harvester. It is observed that by proper selection of parameters, different electrical modes can be driven into degenerate and combination PR by exciting the variable mechanical capacitor over a wide range of frequencies. Simulations further indicate that the proposed 2-DOF system exhibits superior bandwidth and power performance when compared to a 1-DOF system utilizing the same mechanical receiver thereby enabling the use of such PE MDOF systems for broadband energy harvesting with a small mechanical footprint.

\section{Methods}

\subsection{Lumped parameter modelling of a capacitively coupled electromechanical resonator with $n$-degrees of freedom}

A lumped parameter 1D model previously developed for a 1-DOF CPUT [24] is modified to include the proposed PE MDOF capacitive electromechanical resonator and is shown in figure 1 . The MDOF system consists of a mechanical parallel plate resonator with the moving plate of known mass $m$, stiffness $k$ and damping $b$, that also acts as a time-varying capacitor $C_{\mathrm{var}}$ in the electrical domain. This is connected in series with an electrical resonator having $n$ DOF, where $n$ is the number of shunted LC branches and a load resistance $R_{\mathrm{L}}$. In the absence of $C_{\mathrm{var}}$, the resonant frequency $f_{i}$ of the $i$ th LC branch only depends on the values of $L_{i}$ and $C_{i}$. However, in the case of the MDOF system, each LC branch is capacitively coupled to all of the other branches through the time-varying capacitor. A mechanical excitation applied to the moving plate changes the instantaneous value of $C_{\mathrm{var}}$ and perturbs the resonance frequency of all $n$ branches. If a harmonic excitation of frequency $2 f_{i}$ is applied on the moving plate, the $i$ th branch of the electrical resonator can be driven into degenerate $\mathrm{PR}$, with the amplitude of voltage across the load resistance $R_{\mathrm{L}}$ only limited by the inherent nonlinearities [15]. The initial perturbation required for the onset of PR can be satisfied by the thermal noise present in the electrical resonator. Additionally, the system can be driven into combination PR by exciting the plate around $f_{i}+f_{j}$ where $i$ and $j$ represent two different LC branches of the electrical resonator [28]. Thus, increasing the number of LC branches allows for a larger number of frequency bands in ${ }^{3}$ Note that henceforth, degrees of freedom (DOF) refer to the number of elec-
trical modes unless mentioned otherwise. 


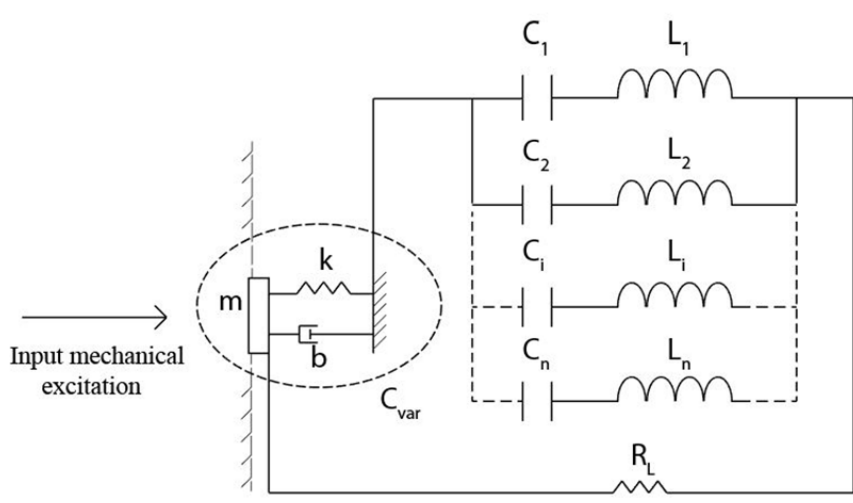

Figure 1. A 1D lumped parameter schematic of a capacitive electromechanical resonator with $n$-DOF.

which the system can be driven into degenerate and combination PR, thereby dramatically increasing the bandwidth while using a single mechanical resonating element.

\subsection{Implementation of model in Simulink}

The MDOF system can also be represented by a set of coupled nonlinear differential equations that can be solved iteratively in the time domain by a commercial solver such as Simulink (MathWorks, Natick MA). This enables one to simulate its operation for various input forcing conditions, electrical parameters and designs. The governing equations for the systems are:

$$
\begin{gathered}
{\left[\frac{\mathrm{d}^{2}}{\mathrm{~d} t^{2}}+\frac{b}{m} \frac{\mathrm{d}}{\mathrm{d} t}+\frac{k}{m}\right] x=\frac{F_{0}}{m} \sin \left(\omega_{\text {in }} t\right)+\frac{\varepsilon_{0} A}{2 m} \frac{V_{\mathrm{c}}^{2}}{\left(d_{0}-x\right)^{2}}} \\
V_{\mathrm{c}}+i\left[R_{\mathrm{L}}+\mathcal{F}^{-1}\{\tilde{Z}(\omega)\}\right]=0 \\
i=\frac{\mathrm{d}\left[\frac{\varepsilon_{0} A}{\left(d_{0}-x\right)} V_{\mathrm{c}}\right]}{\mathrm{d} t} .
\end{gathered}
$$

Here, equation (1) represents the dynamics of the mechanical parallel plate resonator with electrical and mechanical force inputs, equation (2) is Kirchhoff's voltage law applied to the system and equation (3) represents the relationship between the current in the circuit and the voltage across the variable capacitor. The parallel plate displacement is given by $x$ and the voltage across the variable capacitor is given by $V_{\mathrm{c}}$. The angular frequency of the input mechanical excitation is $\omega_{\text {in }}$ and the load resistance is $R_{\mathrm{L}}$. The impedance of the $n$ shunted electrical oscillators is represented by $\tilde{Z}(\omega)$ where,

$$
\frac{1}{\tilde{Z}(\omega)}=\frac{1}{j \omega L_{1}+\frac{1}{j \omega C_{1}}}+\frac{1}{j \omega L_{2}+\frac{1}{j \omega C_{2}}}+\ldots+\frac{1}{j \omega L_{n}+\frac{1}{j \omega C_{n}}} .
$$

Hence, $n$ number of electrical resonators/LC branches can be conveniently expressed in the form of a single complex impedance. In order to solve this system of equations in Simulink, it is necessary to take the inverse Fourier transform

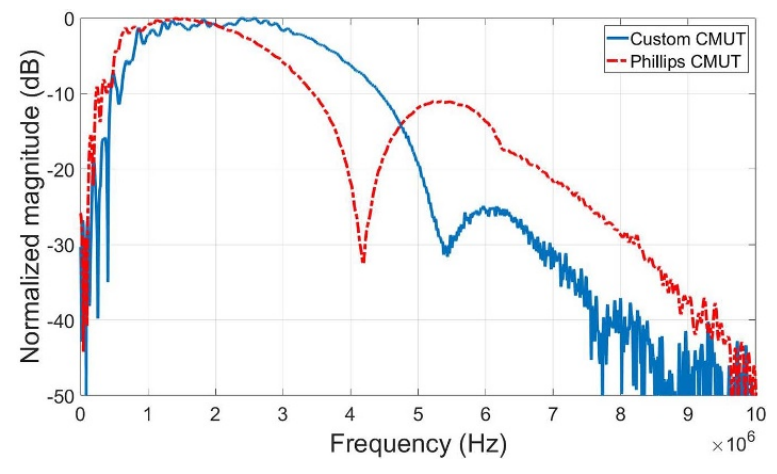

Figure 2. Normalized frequency response of both the custom CMUT and the Phillips CMUT when immersed in Flourinert-FC70: the frequency response is obtained by applying a $100 \mathrm{~ns}$ electrical pulse to the CMUT and recording the output pressure on a broadband hydrophone. The steps involved in the fabrication of a similar device is presented in [30].

(expressed by the $\mathcal{F}^{-1}$ operator in equation 2) of $\tilde{Z}(\omega)$ and express it in the form a finite impulse response filter in the model to keep all the expressions in time domain. The solution of the MDOF system can then be obtained as a function of various parameters such as the input excitation level, forcing frequency etc. The implementation of the model in Simulink is shown in figure $\mathrm{S} 1$ of the supplementary section (available online at stacks.iop.org/SMS/30/045024/mmedia).

\subsection{Experimental demonstration of multiple tunable instability tongues using capacitive micromachined ultrasonic transducers}

Capacitive electromechanical resonators with two electrical DOF are used to experimentally demonstrate that PE MDOF systems can be driven into multiple frequency bands or regions of PR and that these frequencies can be easily tuned. Both the frequency bands and tuning capability are predicted by the model. The first set of proof-of-principle experiments is aimed at demonstrating degenerate and nondegenerate PR in the 2DOF system and its depedence on the level of input excitation. For this experiment, a custom-built capacitive micromachined ultrasonic transducer (CMUT) is used as the mechanical resonating element. A CMUT is a membrane-based MEMS device with a broad bandwidth that is primarily developed and used for medical imaging applications [29]. In this particular experiment, the broadband nature of the CMUT enables us to efficiently excite the system into PR over a wide range of frequencies. The CMUT is ultrasonically excited in Flourinert FC-70 (Sigma-Aldrich), a nonconductive liquid with a charactersitic acoustic impedance similar to that of water. Figure 2 shows the pressure output spectrum of the CMUT in FC70. Note that the mechanical response of the CMUT is quite damped, with a $-3 \mathrm{~dB}$ bandwidth of roughly $2.5 \mathrm{MHz}$ around $2.4 \mathrm{MHz}$. This is significant as the electrical resonator-based approach here enables PR without the need for lightly damped mechanical resonators or experiments in vacuum chambers [16]. 


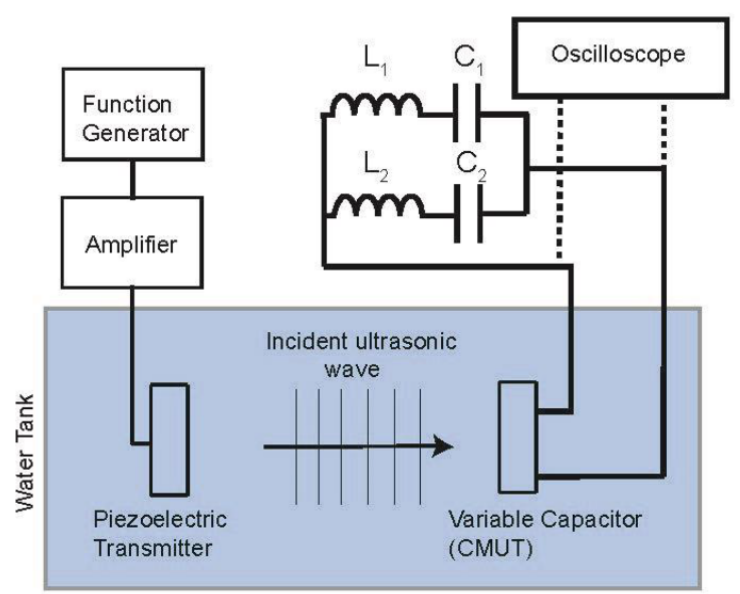

Figure 3. Schematic of the experimental setup used to demonstrate multiple tunable regions of PR in a 2-DOF capactive electromechanical resonator.

The static capacitance $C_{\text {var }}$ of the custom CMUT is measured in FC-70 using a network analyzer and is found to be $38 \mathrm{pF}$. The CMUT is connected to an electrical resonator having two LC branches and the values $L_{1}(1.34 \mathrm{mH}), C_{1}(500 \mathrm{pF})$, $L_{2}(1.55 \mathrm{mH})$ and $C_{2}(19 \mathrm{pF})$ are chosen such that the undisturbed linear resonance frequencies of the system are $560 \mathrm{kHz}$ $\left(f_{1}\right)$ and $1.23 \mathrm{MHz}\left(f_{2}\right)$. Theoretically it is possible to reduce the spacing between the two electrical resonance frequencies $f_{1}$ and $f_{2}$, however calcuations reveal that the values of inductance required for such an implementation are impractically large, given the low value of $\mathrm{C}_{\mathrm{var}}$. (refer to supplementary material for more details on calculation and optimization of capactiances and inductances). Note that no DC bias is applied to the CMUT capacitance when operated in this configuration. A piezoelectric transducer (Olympus A303S/306S-SU) connected to a signal generator and an RF amplifier is used to mechanically excite the CMUT. The narrowband nature of the piezoelectric transducer requires the use of two different transducers at $1 \mathrm{MHz}$ and $2.25 \mathrm{MHz}$ to successfully excite the system into PR in the different instability regions. An oscilloscope (Tektronix TDS5054B) is used to measure the voltage across $C_{\mathrm{var}}$. The experimental setup is shown in figure 3 .

In a second set of experiments aimed at demonstrating tunability of the PR instability tongues, a commercially available CMUT device (Philips innovations) operating at $1.5 \mathrm{MHz}$ with a $-3 \mathrm{~dB}$ bandwidth of $1.97 \mathrm{MHz}$ is used as the mechanical resonating element in a similar configuration to that shown in figure 4 . These devices are particularly suitable for this purpose because their large static capacitance of $353 \mathrm{pF}$ (approximately $10 \times$ larger than the custom CMUT) enables closely spaced electrical resonance frequencies using practical, off-the-shelf inductors. The values of $L_{1}, C_{1}, L_{2}$ and $C_{2}$ are re-calculated using the new value of $C_{\text {var }}$ to realize electrical resonance frequencies $f_{1}$ and $f_{2}$ at $458 \mathrm{kHz}$ and $587 \mathrm{kHz}$ respectively. The value of inductor $L_{2}$ is also varied to demonstrate easy electrical tuning of the resonance frequencies which in turn leads to superior control over the location of the PR instability tongues when compared to purely

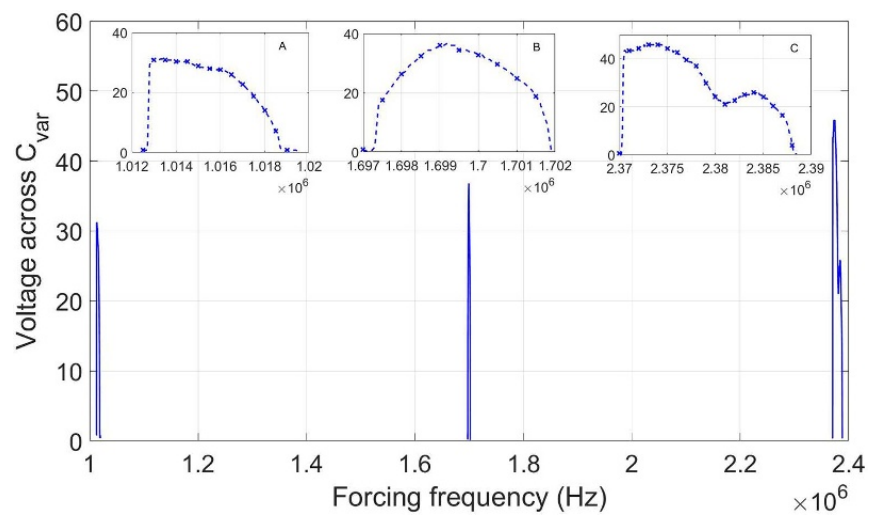

Figure 4. Experimentally measured frequency response of the custom CMUT based 2-DOF electromechanical resonator displaying three distinct regions of PR centered at forcing frequencies of $1.016 \mathrm{MHz}, 1.699 \mathrm{MHz}$ and $2.378 \mathrm{MHz}$.

Table 1. Comparison between custom CMUT and the commercial CMUT used in the experiments along with circuit components used to create the 2-DOF system.

\begin{tabular}{lll}
\hline System properties & Custom CMUT & Philips CMUT \\
\hline $\begin{array}{l}\text { Membrane } \\
\text { dimensions }\end{array}$ & $70 \mu \mathrm{m} \times 70 \mu \mathrm{m}$ & $100 \mu \mathrm{m}$ (radius) \\
$\begin{array}{l}\text { Center frequency } \\
\text { Mechanical Q-factor }\end{array}$ & $2.4 \mathrm{MHz}$ & $1.5 \mathrm{MHz}$ \\
$\begin{array}{l}\text { No. of membranes } \\
\text { Static capacitance }\end{array}$ & 306 & 0.602 \\
$\begin{array}{l}C_{\text {var }} \text { ) } \\
\text { Inductor 1 }\left(L_{1}\right)\end{array}$ & $1.34 \mathrm{pF}$ & 672 \\
$\begin{array}{l}\text { Inductor 2 }\left(L_{2}\right) \\
\text { Capacitor 1 }\left(C_{1}\right)\end{array}$ & $1.55 \mathrm{mH}$ & $353 \mathrm{pF}$ \\
$\begin{array}{l}\text { Capacitor 2 }\left(C_{2}\right) \\
\text { Resonance }\end{array}$ & $19 \mathrm{pF}$ & $4.8 \mathrm{mH}$ \\
$\begin{array}{l}\text { frequency 1 }\left(f_{1}\right) \\
\text { (predicted/measured) }\end{array}$ & $560 \mathrm{kHz} / 508.9 \mathrm{kHz}$ & $460 \mu \mathrm{H}$ \\
$\begin{array}{l}\text { Resonance } \\
\text { frequency 2 }\left(f_{2}\right)\end{array}$ & $1.23 \mathrm{MHz} / 1.189 \mathrm{MHz}$ \\
(predicted/measured) & & $600 \mathrm{kHz} / 587 \mathrm{kHz}$ \\
\hline
\end{tabular}

mechanical PE systems. The main differences between the custom-made and commercial CMUTs along with the circuit components used for the two sets of experiments are highlighted in table 1 in comparison with the model predictions.

\section{Results and discussion}

3.1. Experimental results: existence of multiple regions of $P R$ in systems with high mechanical damping and dependency on excitation level

The frequency response of the 2-DOF system is determined by applying an incident ultrasonic excitation and measuring the voltage across the variable capacitor. The load resistance is set to zero as a large internal resistance $(\approx 25 \Omega)$ in the CMUT contributes to a significant increase in the forcing threshold required to drive the system into PR and any 
additional resistance would increase this threshold and reduce the bandwidth. The input voltage to the piezoelectric transducer is adjusted such that the ultrasonic excitation is sufficient to drive the system into PR. The measured linear resonance frequencies $f_{1}$ and $f_{2}$ are found to be $508.9 \mathrm{kHz}$ and 1.189 $\mathrm{MHz}$ respectively and this minor shift from the theoretically calculated values can be attributed to differences between the rated and actual values of the coil wound inductors as well as parasitics in the circuit. The voltage across the CMUT plotted as a function of the forcing frequency (figure 4) shows that there are three regions where the system exhibits a large response and each of them corresponds to an LC branch driven into PR. A closer observation of first of these regions (region A) reveals an asymmetric instability tongue associated with the primary PR of the lower LC branch. This is confirmed by a sharp increase in the voltage across the CMUT as the forcing frequency crosses the boundary of the instability tongue centered on $2 f_{1}$. A spectral analysis of the recorded output voltage also reveals that the primary frequency component is half of the forcing frequency, additionally confirming that the system is PE in this region. Similarly, region C corresponds to the instability tongue associated with the primary PR of the upper LC branch and is centered approximately on $2 f_{2}$. A third region where the 2-DOF system is driven into PR occurs at region $\mathrm{B}$ and is a result of the combination resonance associated with the interaction of the two LC branches. This interaction is unique to MDOF PE systems and provides an additional region of system response that is not seen in linear MDOF systems. The instability tongue for region $\mathrm{B}$ is centered on $f_{1}+f_{2}$ and the primary frequency components of the recorded output voltage are around $f_{1}$ and $f_{2}$. As expected, the voltage is negligible at forcing frequencies outside the three instability tongues as the system cannot be driven into PR.

The bandwidth of the parametric instability tongue has previously been shown to depend strongly on the level of forcing [26]. To observe the effect of an increased input force, the voltage across the CMUT capacitance is recorded for different levels of piezoelectric excitation. Since two different transducers are used to excite the CMUT at different frequencies, the differences between the input voltages applied to the transducer must only be compared within each instability tongue. Figure 5 shows the effect of increasing the forcing level on the bandwidth of the three regions of PR. In each case, the width of the instability tongue increases with increasing input voltage level. The bandwidth can be further increased by reducing the internal resistance present in the CMUT capacitance. Doing so also ensures that maximum power is delivered to the load resistance enabling efficient operation at lower input excitation levels.

\subsection{Experimental results: electrical tuning of $P R$ frequencies}

As previously mentioned, the low static capacitance of the custom-made CMUT requires the use of very large inductances in order to obtain closely spaced electrical resonances. This limitation can be compensated to some degree by using a CMUT with a larger static capacitance. The custom-made CMUT used previously is replaced by the Philips CMUT with
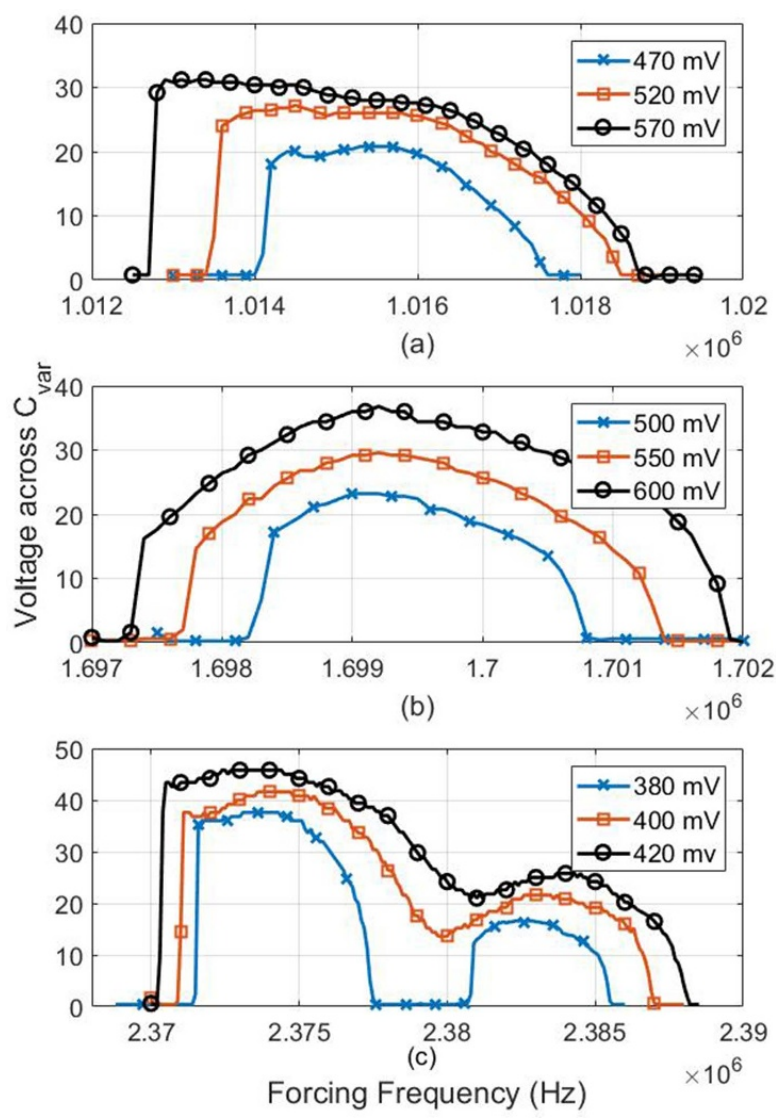

Figure 5. Effect of input forcing level on the bandwidth of the 2-DOF system. (a)-(c) Correspond to the instability tongues centered at frequencies $2 f_{1}, f_{1}+f_{2}$ and $2 f_{2}$ respectively.

the intention of reducing the spacing between the different instability tongues and demonstrating the tunable nature of the electrical resonances. The optimal circuit parameters are calculated and a new 2-DOF system is realized using practically achievable inductance and capacitance values. Once again, the measured resonance frequencies $f_{1}(458 \mathrm{kHz})$ and $f_{2}(587 \mathrm{kHz})$ closely match the predictions from the theoretical model, with negligible variations caused by parasitics in the circuit. The closely spaced nature of the resonance frequencies enables the excitation of all three instability tongues with a single $1 \mathrm{MHz}$ piezoelectric transducer (figure 6). The location of each of the instability tongues is consistent with the previous results and is centered approximately around $2 f_{1}, 2 f_{2}$ and $f_{1}+f_{2}$ for the primary PRs and combination PR respectively. The variations in the amplitude of voltage across $C_{\mathrm{var}}$ and the shape of the instability tongues when compared to the results obtained with the custom-made CMUT can be attributed to differences in forcing levels and mechanical properties of the CMUTs. The value of $L_{2}$ is also reduced by $10 \mu \mathrm{H}$ and the frequency response of the system is re-measured to demonstrate that the LC resonators can be easily tuned to adjust the location of the instability tongues (dashed line). As further validation of the model, it is noted that the movement of the instability tongues toward the right is in agreement with the theoretical prediction of a $6 \mathrm{kHz}$ and $4 \mathrm{kHz}$ increase in the two linear resonance frequencies, respectively. It is worth noting 


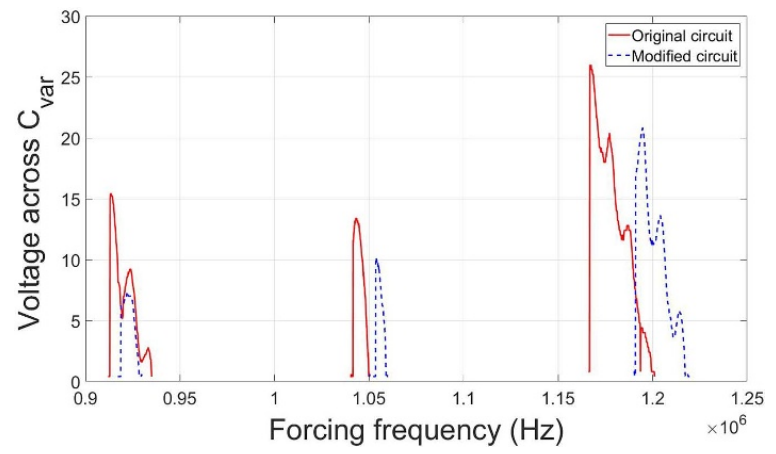

Figure 6. Measured frequency response of the 2-DOF system with a commercially available CMUT as the mechanical resonating element (solid line). Note the reduction in frequency spacing between the instability tongues as compared to figure 5 . The dashed lines represent the frequency response of the system when $L_{2}$ is reduced by $10 \mu \mathrm{H}$.

that the realization of such closely spaced tunable instability tongues in purely mechanical PE systems is extremely challenging to achieve as the mechanical structure itself must be modified in order to move around the vibrational modes. Hence, the MDOF electrical resonator-based approach allows for a greater degree of flexibility while designing PE capacitive energy harvesting systems.

Thus, by making use of a capacitive electromechanical resonator with MDOF, it is possible to realize a PE energy harvesting device with a significantly larger frequency band of operation. The electrical resonance frequencies can be easily adjusted, allowing the device to operate in frequency bands containing the largest input excitations. Experimental results indicate that while the design of the mechanical resonating element can be flexible, having a large static capacitance enables the realization of closely spaced resonance frequencies without the need for very large inductors. Finally, the use of a damped mechanical element to excite the system into different regions of PR demonstrates the relative independence of the system to environmental and mechanical damping thereby overcoming a common limitation of purely mechanical PE energy harvesting systems.

\subsection{Design and simulation of a low frequency elastic wave energy harvester}

To verify that the concept of MDOF can also be applied to energy harvesting, a PE capacitive energy harvester operating at a lower frequency than the CMUT used in the experiment is designed and simulated in Simulink [24]. Ambient vibration energy harvesting systems commonly operate at frequencies below $100 \mathrm{~Hz}$, however synthesizing passive LC resonators at such frequencies requires the use of impractically large inductors. Here, elastic wave energy harvesting [31-35] is specifically chosen as the target application as the MDOF approach is best suited for operation in the $>40 \mathrm{kHz}$ frequency band.

The proposed system consists of a mechanical parallel plate resonator which is modeled as a generic mass-spring-dashpot with a resonance frequency of $50 \mathrm{kHz}$ and connected to a
Table 2. List of parameters used for the simulation of the low frequency energy harvester.

\begin{tabular}{lll}
\hline Symbol & Parameter & Value \\
\hline$m$ & Mass of plate & $101.3 \mathrm{~g}$ \\
$k$ & Stiffness of plate & $1 \times 10^{10} \mathrm{~N} \mathrm{~m}$ \\
$b$ & Damping in plate & $3000 \mathrm{~N} \mathrm{~s} \mathrm{~m}^{-1}$ \\
$g$ & Equivalent gap & $80 \mathrm{~nm}$ \\
$A$ & Equivalent area & $6.15 \times 10^{-4} \mathrm{~m}^{2}$ \\
$L_{1}$ & Inductor 1 & $83 \mathrm{mH}$ \\
$L_{2}$ & Inductor 2 & $688 \mu \mathrm{H}$ \\
$C_{1}$ & Capacitor 1 & $0.482 \mathrm{nF}$ \\
$C_{2}$ & Capacitor 2 & $0.478 \mu \mathrm{F}$ \\
$C_{\text {var }}$ & Parallel plate capacitance & $68.14 \mathrm{nF}$ \\
$R_{\mathrm{L}}$ & Load resistance & $5 \Omega$ \\
\hline
\end{tabular}

2-DOF electrical resonator. The mechanical and electrical parameters used in the model are listed in table 2 . The values of the electrical components are chosen such that the lower and upper resonance frequencies of the electrical resonator are at $48 \mathrm{kHz}\left(f_{1}^{\prime}\right)$ and $52 \mathrm{kHz}\left(f_{2}^{\prime}\right)$, respectively. The input is provided in the form of a base excitation to mimic conditions present in a typical elastic wave energy harvesting scenario. Additionally, a $1 \mathrm{~V}$ unipolar electrical pulse with a width of $4 \mu \mathrm{s}$ is applied to the circuit at $t=0$, in order to provide the initial conditions required for $\mathrm{PR}$ in the system.

Three different excitations of $0.546 \mathrm{~mm} \mathrm{~s}^{-1}, 0.703 \mathrm{~mm} \mathrm{~s}^{-1}$ and $0.858 \mathrm{~mm} \mathrm{~s}^{-1}$ are applied to the harvester and the voltage across the load resistance as a function of the forcing frequency is plotted in figure 7(a). Note that these forcing levels and frequencies are typical to previously reported elastic wave energy harvesting systems [36, 37]. Parametric instability tongues are observed around $2 f_{1}^{\prime}, 2 f_{2}^{\prime}$ and $f_{1}^{\prime}+f_{2}^{\prime}$ due to primary and combination PRs. Similar to the experimentally observed trends, the width of the instability tongue increases with input acceleration. Due to the closely spaced resonance frequencies $f_{1}^{\prime}$ and $f_{2}^{\prime}$, a high level of base excitation $\left(0.858 \mathrm{~mm} \mathrm{~s}^{-1}\right)$ increases the bandwidth of each of the instability tongues such that they overlap with each other creating a continuous band of operation. Thus, the system directly transitions from one instability tongue to another while constantly maintaining a large output response to the excitation.

In order to highlight the advantage of MDOF, the performance of the 2-DOF PE capacitive energy harvester is compared with two 1-DOF PE harvesters having electrical resonance frequencies at $24 \mathrm{kHz}$ and $26 \mathrm{kHz}$. The 1-DOF systems utilize the same mechanical resonating element as that used in the 2DOF system and is connected in series with the appropriate inductor and $5 \Omega$ load resistance. The simulated performance of the three systems for an input excitation of $0.703 \mathrm{~mm} \mathrm{~s}^{-1}$ is shown in figure 7(b). It is observed that the 2-DOF system has an operational band spanning approximately $5250 \mathrm{~Hz}$ whereas the 1-DOF systems span $4700 \mathrm{~Hz}(24 \mathrm{kHz})$ and $4780 \mathrm{~Hz}$ $(26 \mathrm{kHz})$ for the same input excitation. Furthermore, a peak voltage of $0.5 \mathrm{~V}$ across the $5 \Omega$ load resistance of the 2-DOF system exceeds that of either of the 1-DOF systems $(0.45 \mathrm{~V})$. Note that the operational bandwidth of the 2-DOF system can be further increased by optimizing the electrical parameters 


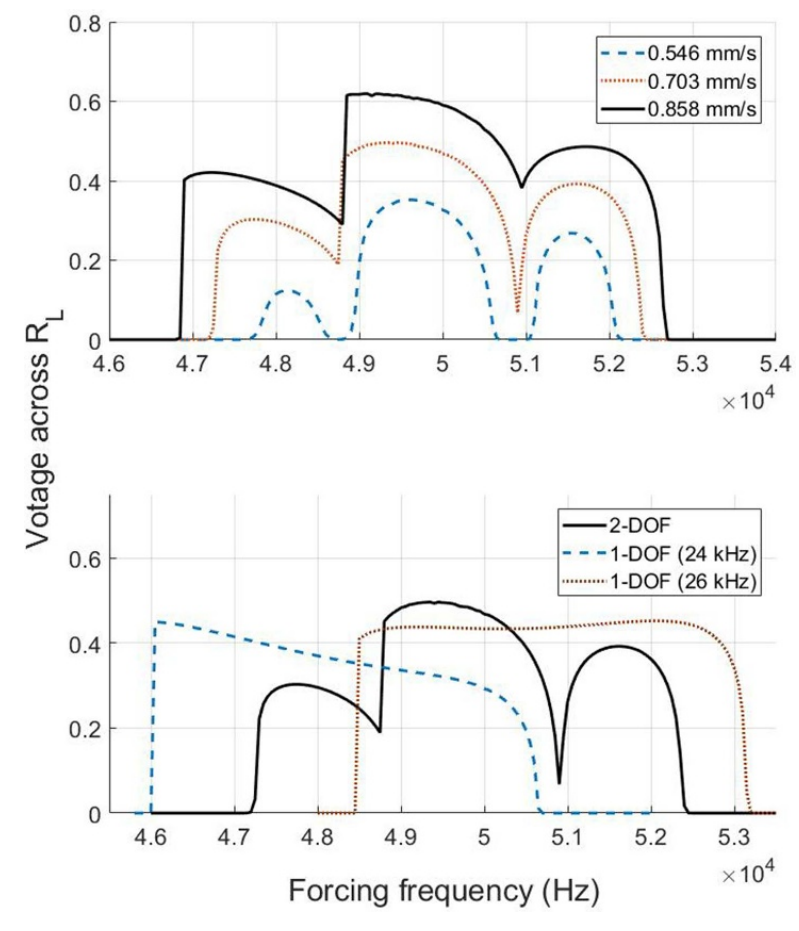

Figure 7. (a) Simulated voltage across the load resistance of a 2-DOF PE capacitive energy harvester for three different values of input base excitation. (b) Comparison of the 2-DOF system with two 1-DOF systems having resonance frequencies at $5 \mathrm{kHz}$ and $6 \mathrm{kHz}$ for an input excitation of $0.703 \mathrm{mms} \mathrm{s}^{-1}$.

or by adding additional LC resonators (refer to supplementary material). Thus, the simulations build on the experimental demonstration by indicating that adding additional DOF to the electrical resonator and tuning the resonance frequencies such that they are closely spaced can enable significant increase in the bandwidth of the low frequency PE energy harvester at practical excitation levels.

\section{Conclusions}

In summary, this work shows that multiple instability tongues associated with degenerate and combination PRs can be excited in an electromechanical system with MDOF. This approach makes it possible to design and implement PR based electromechanical devices operating over a wider range of frequencies and operating conditions. Experiments performed using CMUTs in immersion demonstrated that a 2-DOF capacitive electromechanical resonator can be driven into three distinct regions of PR. The frequency band in which the device operates can be easily tuned by simply modifying the electrical circuit parameters while maintaining the same mechanical resonator. Since the threshold for PR is primarily dependent on the resistance in the electrical circuit, the system can be operated even in damped environments without the need for a high$Q$ mechanical resonator.

Simulations performed using a 1D lumped parameter model show that the MDOF PE capacitive energy harvester can be utilized for broadband elastic wave energy harvesting in the $>40 \mathrm{kHz}$ frequency regime. Closely spaced electrical resonators cause the instability tongues to merge and form a continuous region of response for energy harvesting at high excitation levels. It is also seen that the 2-DOF PE energy harvester has superior bandwidth and peak output power when compared to a 1D system using the same mechanical resonator. Moreover, a DC voltage or electret to bias the capacitive energy harvester is not required in the proposed system. Further improvements in bandwidth can be practically achieved by including additional electrical resonators with optimized resonance frequencies.

Future work includes the fabrication and characterization of the low frequency energy harvester and further investigations to understand the parameters affecting the bandwidth and minimum forcing threshold levels of each of the PE modes.

\section{Acknowledgments}

This work was supported by the ECCS division of the National Science Foundation through NSF ECCS Award Nos. 1936776 and 1829821. Device fabrication was performed in part at the Georgia Tech Institute for Electronics and Nanotechnology, a member of the National Nanotechnology Coordinated Infrastructure (NNCI), which is supported by the National Science Foundation (Grant ECCS-1542174). The authors would also like to thank Dr Evren Arkan for fabricating the custom CMUTs used in the experiments.

\section{ORCID iD}

Sushruta Surappa (D) https://orcid.org/0000-0002-9415-0434

\section{References}

[1] Tran N, Ghayesh M H and Arjomandi M 2018 Ambient vibration energy harvesters: a review on nonlinear techniques for performance enhancement Int. J. Eng. Sci. $127162-85$

[2] Daqaq M F, Stabler C, Qaroush Y and Seuaciuc-Osório T 2009 Investigation of power harvesting via parametric excitations J. Intell. Mater. Syst. Struct. 20 545-57

[3] Alevras P, Theodossiades S and Rahnejat H 2017 Broadband energy harvesting from parametric vibrations of a class of nonlinear Mathieu systems Appl. Phys. Lett. 110233901

[4] Yildirim T, Ghayesh M H, Li W and Alici G 2016 Design and development of a parametrically excited nonlinear energy harvester Energy Convers. Manage. 126 247-55

[5] Garg A and Dwivedy S K 2020 Piezoelectric energy harvester under parametric excitation: a theoretical and experimental investigation J. Intell. Mater. Syst. Struct. 31 612-31

[6] Jia Y, Yan J, Soga K and Seshia A A 2014 A parametrically excited vibration energy harvester J. Intell. Mater. Syst. Struct. 25 278-89

[7] Yang W and Towfighian S 2019 A parametric resonator with low threshold excitation for vibration energy harvesting $J$. Sound Vib. 446 129-43

[8] Jia Y and Seshia A A 2014 An auto-parametrically excited vibration energy harvester Sensors Actuators A 220 69-75

[9] Jia Y, Du S, Arroyo E and Seshia A A 2018 Autoparametric resonance in a piezoelectric MEMS vibration energy harvester 2018 IEEE Micro Electro Mechanical Systems (MEMS) pp 226-9 
[10] Kuang Y and Zhu M 2019 Parametrically excited nonlinear magnetic rolling pendulum for broadband energy harvesting Appl. Phys. Lett. 114203903

[11] Kuang Y, Hide R and Zhu M 2019 Broadband energy harvesting by nonlinear magnetic rolling pendulum with subharmonic resonance Appl. Energy 255113822

[12] Nabholz U, Lamprecht L, Mehner J E, Zimmermann A and Degenfeld-Schonburg P 2020 Parametric amplification of broadband vibrational energy harvesters for energy-autonomous sensors enabled by field-induced striction Mech. Syst. Signal Process. 139106642

[13] Jia Y, Yan J, Soga K and Seshia A A 2013 Parametrically excited MEMS vibration energy harvesters with design approaches to overcome the initiation threshold amplitude J. Micromech. Microeng. 23114007

[14] Jia Y, Yan J, Soga K and Seshia A A 2014 Parametric resonance for vibration energy harvesting with design techniques to passively reduce the initiation threshold amplitude Smart Mater. Struct. 23065011

[15] Zaghari B, Kniffka T, Levett C and Rustighi E 2019 Parametrically excited nonlinear two-degree-of-freedom electromechanical systems

[16] Wallin C B et al 2018 Nondegenerate parametric resonance in large ensembles of coupled micromechanical cantilevers with varying natural frequencies Phys. Rev. Lett. 121264301

[17] Dolev A and Bucher I 2018 Dual frequency parametric excitation of a nonlinear, multi degree of freedom mechanical amplifier with electronically modified topology J. Sound Vib. 419 420-35

[18] Welte J, Kniffka T J and Ecker H 2013 Parametric excitation in a two degree of freedom MEMS system Shock Vib. 20 1113-24

[19] Yildirim T, Zhang J, Sun S, Alici G, Zhang S and Li W 2017 Design of an enhanced wideband energy harvester using a parametrically excited array J. Sound Vib. 410 416-28

[20] Seshia A A, Du S and Jia Y 2016 Twenty-eight orders of parametric resonance in a microelectromechanical device for multi-band vibration energy harvesting Sci. Rep. 630167

[21] Jia Y, Yan J, Soga K and Seshia A A 2013 Multi-frequency operation of a MEMS vibration energy harvester by accessing five orders of parametric resonance $J$. Phys.: Conf. Ser. 476012126

[22] Yildirim T, Ghayesh M H, Searle T, Li W and Alici G 2017 A parametrically broadband nonlinear energy harvester $J$. Energy Resour. Technol. 139032001

[23] Yildirim T, Ghayesh M H, Li W and Alici G 2017 A nonlinearly broadband tuneable energy harvester J. Dyn. Syst. Meas. Contr. 139011008
[24] Surappa S, Tao M and Degertekin F L 2018 Analysis and design of capacitive parametric ultrasonic transducers for efficient ultrasonic power transfer based on a 1D lumped model IEEE Trans. Ultrason. Ferroelectr. Freq. Control $652103-12$

[25] Caldwell N B and Daqaq M F 2017 Exploiting the principle parametric resonance of an electric oscillator for vibratory energy harvesting Appl. Phys. Lett. 110093903

[26] Surappa S and Degertekin F L 2020 Characterization of a parametric resonance based capacitive ultrasonic transducer in air for acoustic power transfer and sensing Sensors Actuators A 303111863

[27] Surappa S, Satir S and Levent Degertekin F 2017 A capacitive ultrasonic transducer based on parametric resonance Appl. Phys. Lett. 111043503

[28] Kniffka T J, Mace B R, Ecker H and Halkyard R 2016 Studies of parametrically excited non-linear MDOF systems at parametric resonances J. Phys.: Conf. Ser. 744012126

[29] Oralkan Ö, Ergun A S, Johnson J A, Karaman M, Demirci U, Kaviani K, Lee T H and Khuri-Yakub B T 2002 Capacitive micromachined ultrasonic transducers: next-generation arrays for acoustic imaging? IEEE Trans. Ultrason. Ferroelectr. Freq. Control 49 1596-610

[30] Pirouz A and Degertekin F L 2018 Low temperature CMUT fabrication process with dielectric lift-off membrane support for improved reliability J. Micromech. Microeng. 28085006

[31] Chen Z, Guo B, Yang Y and Cheng C 2014

Metamaterials-based enhanced energy harvesting: a review Physica B 438 1-8

[32] Tan T, Yan Z, Zou H, Ma K, Liu F, Zhao L, Peng Z and Zhang W 2019 Renewable energy harvesting and absorbing via multi-scale metamaterial systems for internet of things Appl. Energy 254113717

[33] Carrara M, Cacan M R, Toussaint J, Leamy M J, Ruzzene M and Erturk A 2013 Metamaterial-inspired structures and concepts for elastoacoustic wave energy harvesting Smart Mater. Struct. 22065004

[34] Zareei A, Darabi A, Leamy M J and Alam M-R 2018 Continuous profile flexural GRIN lens: focusing and harvesting flexural waves Appl. Phys. Lett. 112023901

[35] Lee T-G, Jo S H, Seung H M, Kim S W, Kim E J, Youn B D, Nahm S and Kim M 2020 Enhanced energy transfer and conversion for high performance phononic crystal-assisted elastic wave energy harvesting Nano Energy 78105226

[36] Tol S, Degertekin F L and Erturk A 2017 Phononic crystal Luneburg lens for omnidirectional elastic wave focusing and energy harvesting Appl. Phys. Lett. 111013503

[37] Tol S, Degertekin F L and Erturk A 2019 3D-printed phononic crystal lens for elastic wave focusing and energy harvesting Addit. Manuf. 29100780 